\title{
Research on the Artistic Conception of Multimedia-Assisted Ancient Poetry Based on AI Technology
}

\author{
Yanna Jiao \\ Basic Theory Department, Henan Industry and Trade Vocational College, Zhengzhou 450000, China \\ Correspondence should be addressed to Yanna Jiao; jiaoyanna@hngm.edu.cn
}

Received 7 December 2021; Revised 23 December 2021; Accepted 27 December 2021; Published 25 January 2022

Academic Editor: Tongguang $\mathrm{Ni}$

Copyright (c) 2022 Yanna Jiao. This is an open access article distributed under the Creative Commons Attribution License, which permits unrestricted use, distribution, and reproduction in any medium, provided the original work is properly cited.

\begin{abstract}
In order to improve the visual display effect of the artistic conception of ancient poems, this paper combines AI technology to construct a multimedia-assisted artistic conception creation system of ancient poetry and combines probabilistic latent semantic analysis to analyze the semantic artistic conception of ancient poetry. Moreover, with the support of multimedia simulation technology, this paper improves the multimedia teaching mode to improve the effect of multimedia-assisted ancient poetry teaching. In addition, this paper constructs a multimedia-assisted artistic conception creation system of ancient poetry based on AI technology and designs experiments to conduct system performance research. The experimental research results show that the multimedia-assisted artistic conception creation system of ancient poetry proposed in this paper has a good teaching effect and has an important role in promoting the study of the artistic conception of ancient poetry.
\end{abstract}

\section{Introduction}

It is difficult to understand the author's thoughts and feelings and experience the beauty of the artistic conception of poetry. When teaching ancient poems to students, the teacher needs to combine the cognitive characteristics of different ages to make students fully understand the writing mentality and historical background of the poet during the creation period, so as to induce students' interest in learning ancient poems. Therefore, before each study of an ancient poem, we will arrange a certain amount of spare time for students to go online and use network technology to find, view, and collect relevant information to understand the author's profile, historical background, and corresponding introduction of the poem, so that students have a rough understanding of the poems they have learned and lay a certain teaching foundation.

In the current Chinese teaching in my country, whether it is before or after the release of the new curriculum standard reform, ancient poetry plays a very important role in Chinese teaching at the university level. The reason is that, compared with modern poems and essays, ancient poems have extraordinary significance. Ancient poetry contains a wealth of traditional culture, and through the study of ancient poetry, college students can effectively improve their language expression ability and understand the many situations of ancient society. The improvement that this brings to the students is an improvement in comprehensive literacy, not a unilateral improvement. Especially in recent years, college Chinese textbooks have continued to change, and the proportion of ancient poems in them is increasing, which also proves the importance of ancient poems in the current college Chinese teaching. Multimedia technology is an innovative teaching model that emerged after informatization has penetrated into teaching, which is very popular among students. In addition, it can effectively improve the quality of teaching and can also fully stimulate students' interest in learning. In the current college Chinese ancient poetry teaching, the use of multimedia technology will inevitably improve the teaching effect.

Although the ancient poems at the university stage are relatively simple ancient poems, and some can even be understood in the vernacular way, in the process of teaching, because the college students are younger, they are understanding a lot. In the process of the content, due to insufficient cognitive level and insufficient life experience, there 
will be more serious problems in understanding. For many ancient poems, college students cannot understand the meaning of ancient poems, not to mention the rich thoughts and feelings contained in them. Therefore, in the process of teaching ancient poetry, teachers need to use their own teaching strategies to help students enter a specific teaching situation and guide students to understand the meaning of poetry. To understand the text in ancient poems, multimedia will help us a lot in this process. Using multimedia to create teaching situations can not only effectively improve efficiency but also better integrate students into the teaching situation.

Most of the life experiences of ancient poets have many ups and downs, and the works created by these poets in different periods completely express different thoughts and feelings. Therefore, teachers can use this in the process of teaching. The feature allows students to understand the author's life experience first, and then to understand the author's work, so that students will experience the author's mood at the time and have a better understanding of the thoughts and feelings to be expressed in the article. Poets are great. Most, in the process of writing poems, describe their own circumstances or ideals at the time. Therefore, it is more important to understand the circumstances of the author at that time. So the most effective way to help students understand the author is nothing more than multimedia. Teachers can use the Internet to search for the author's life events in detail, highlighting the key points and the events experienced by the author in the process of writing this poem, so that students can understand the poet at that time more clearly.

This paper combines AI technology to improve the multimedia teaching mode and improve the effect of multimedia-assisted ancient poetry teaching, which lays the foundation for the subsequent improvement of the quality of ancient poetry teaching.

\section{Related Work}

A physics-based simulation method simulates the painting process by building models of painting tools such as pens and paper, using image analogy, texture synthesis, and other methods to achieve the artistic effect of virtual reality. Literature [1] proposed a method of simulating hand-painting with style strokes, and literature [2] proposed a virtual brush model (HairyBrush), which can simulate simple ink painting effects. Reference [3] proposes a virtual art pen model based on the elasticity theory, which satisfies the elastic deformation law and models the art pen as an inverted cone to achieve real-time display effects; reference [4] proposes a virtual art pen model. brush model, which can generate more realistic writing effects. Literature [5] proposed a physics-based Mao Fei physical simulation method mainly to simulate the rendering effect. The model proposed in [6] can simulate the effect of ink diffusion on paper. The system developed in [7] can be used to draw trees, and the effect generated by the system is similar to the effect of oil painting. The method proposed in [8] simulates the effect of artistic painting and develops a corresponding system.
In terms of simulation based on painting effects, literature [9] uses the method of image analogy to achieve artistic style drawing. Literature [10] proposes a method of texture synthesis, proposes common textures from artistic paintings, establishes a texture library, and then selects appropriate textures from the texture library to synthesize new ink paintings. Literature [11] designed a paint model that can express a variety of painting forms including traditional Chinese paintings and watercolors. Many scholars are dedicated to studying how to use computers to generate artistic painting effects. Literature [12] proposes a method of synthesizing artistic paintings based on images and has conducted research on computer rendering of artistic paintings. Literature [13] proposed an art painting system simulation rendering algorithm based on texture synthesis. Literature [14] has done research on the rendering effect of art, which is an attempt to realize the style application of animation ink and wash effect. Literature [15] proposes a design method of dynamic artistic conception in order to protect cultural heritage. The 3D mountain rock model constructed in [16] successfully realized the 3D rendering of the art painting simulation effect of the pen and ink method. This method is based on summarizing the shape and distribution of the pen and ink method in the art painting and proposes a fractal modeling method based on the sketch line. Literature [17] designed a form that can automatically generate ancient cultural connotations from pictures in real time. Literature [18] proposes a tool based on the movement trajectory of the artistic pen and the painting material, which can vividly show the process of artistic painting. In recent years, the innovation of computer technology has led to the rapid development of programmable graphics hardware. Programmable graphics software has become an important tool for scholars to conduct research on 3D graphics rendering. Literature [19] proposed the use of programmable graphics hardware to achieve the effect of hand-drawn Chinese painting. In terms of interactive experience, [20] pointed out that, in order to protect art treasures but still allow people to appreciate them, an interactive desktop that can watch artworks at close range is developed. Viewers can achieve partial magnification of the screen through touch points on the desktop. Literature [21] proposed a new method of displaying and restoring ancient famous paintings from a three-dimensional perspective using TIP technology. Now this system can only display animation effects and cannot achieve interaction.

\section{Multimedia Virtual Semantic Extraction of Ancient Poetry}

Probabilistic latent semantic analysis (PLSA) is a statistically significant model proposed by Hofmann, which is mainly used in text classification and natural language research. When researching natural language, it is often necessary to research synonyms. Probabilistic latent semantics is a better way to solve this kind of research problems. By mapping the high-dimensional sparse vector space of the actual observable "document-word" to the low-dimensional latent space, a connection beyond the vocabulary level is established. 
In the PLSA model, we set the document set $D=\left\{d_{1}, d_{2}, \ldots, d_{N}\right\}$ and the word set $W=\left\{w_{1}, w_{2}, \ldots, w_{M}\right\}$, and the $N \times M$ cooccurrence matrix $N$ composed of the document set $D$ and the word set $W$, as shown in

$$
N=\left[\begin{array}{cccc}
n\left(d_{1}, w_{1}\right) & n\left(d_{1}, w_{2}\right) & \cdots & n\left(d_{1}, w_{M}\right) \\
n\left(d_{2}, w_{1}\right) & n\left(d_{2}, w_{2}\right) & \cdots & n\left(d_{2}, w_{M}\right) \\
\vdots & \vdots & \ddots & \vdots \\
n\left(d_{N}, w_{1}\right) & n\left(d_{N}, w_{2}\right) & \cdots & n\left(d_{N}, w_{M}\right)
\end{array}\right] .
$$

Here, $d_{i}$ represents the $i$-th document, $w_{j}$ represents the $j$-th word, and $n\left(d_{i}, w_{j}\right)$ represents the number of times the $j$-th word appears in the $i$-th document. In matrix $N$, each row represents the number of times each word appears in a certain document, and each column represents the number of times a certain word appears in each document. We assume that there is an implicit variable $z$, that is, the subject variable. If $P\left(d_{i}\right)$ is used to indicate the probability of selecting the $i$-th document, $P\left(z_{k} \mid d_{i}\right)$ is the probability of the topic $z_{k}$ appearing in the $i$-th document. $P\left(w_{j} \mid z_{k}\right)$ represents the probability that the word $w_{j}$ appears in the $k$-th topic, and the relationship between the three is shown in Figure 1.

If it is assumed that document $d$ and word $w$ are conditionally independent under topic $z$, formula (2) can be used to express the cooccurrence probability of document $d$ and word $w$.

$$
P\left(d_{i}, w_{j}\right)=P\left(d_{i}\right) P\left(w_{j} \mid d_{i}\right)=P\left(d_{i}\right) \sum_{k=1}^{K} P\left(w_{j} \mid z_{k}\right) P\left(z_{k} \mid d_{i}\right) .
$$

It can be seen from formula (2) that PLSA is actually a mixed model, and there are two model parameters $P(z \mid d)$ and $P(w \mid z)$. Here, $P(z \mid d)$ is the probability distribution of topic $z$ under a given document $d$, and $P(w \mid z)$ is the probability distribution of word $w$ under a given topic $z$. In the PLSA model, the parameters $P(z \mid d)$ and $P(w \mid z)$ are estimated by using the maximum likelihood estimation method to maximize the probability of the generated sample data. The maximum likelihood function is shown in

$$
\begin{aligned}
L & =\sum_{i=1}^{N} \sum_{j=1}^{M} \log P\left(d_{i}, w_{j}\right)^{n\left(d_{i}, w_{j}\right)} \\
& =\sum_{i=1}^{N} \sum_{j=1}^{M} n\left(d_{i}, w_{j}\right) \log P\left(d_{i}, w_{j}\right) \\
& =\sum_{i=1}^{N} n\left(d_{i}\right)\left[\log P\left(d_{i}\right)+\sum_{j=1}^{M} \frac{n\left(d_{i}, w_{j}\right)}{n\left(d_{i}\right)} \log \sum_{k=1}^{K} P\left(w_{j} \mid z_{k}\right) P\left(z_{k} \mid d_{i}\right)\right] .
\end{aligned}
$$

Here, $n\left(d_{i}\right)=\sum_{j=1}^{M} n\left(d_{i}, w_{j}\right)$, and $n\left(d_{i}\right)$ is not model parameters, so the formula can be reduced to

$$
L \propto \sum_{i=1}^{N} \sum_{j=1}^{M} n\left(d_{i}, w_{j}\right) \log \sum_{k=1}^{K} P\left(w_{j} \mid z_{k}\right) P\left(z_{k} \mid d_{i}\right) .
$$

Documents Words

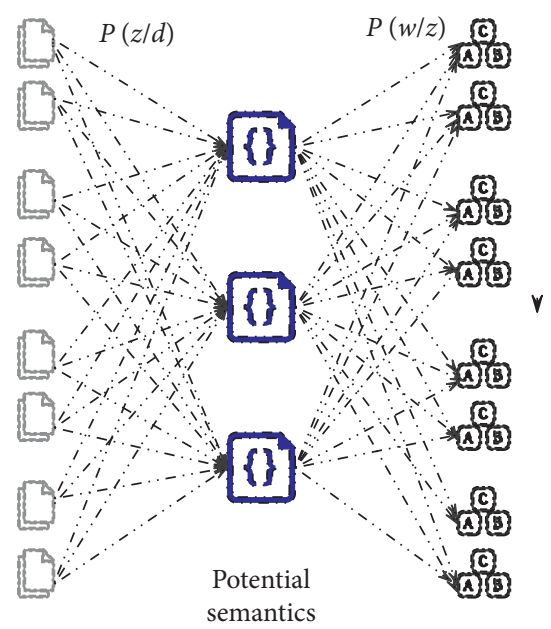

Figure 1: The relationship diagram of "document-latent semantics-word."

It can be seen from formula (4) that $P(z \mid d)$ and $P(w \mid z)$ are the parameters required by the maximum likelihood estimation function. However, since the likelihood function is difficult to maximize, the EM algorithm must be introduced.

The algorithm is an iterative algorithm, divided into two steps. The first step is a step, and what you ask for is expectations. The second step is step, which is the maximum value. The details are shown in formulas (5), (6), and (7).

(1) Step $E$ : the algorithm generates a posterior probability $P\left(z_{k} \mid d_{i}, w_{j}\right)$ for each $\left(d_{i}, w_{j}\right)$ :

$$
P\left(z_{k} \mid d_{i}, w_{j}\right)=\frac{P\left(w_{j} \mid z_{k}\right) P\left(z_{k} \mid d_{i}\right)}{\sum_{k=1}^{K} P\left(w_{j} \mid z_{k}\right) P\left(z_{k} \mid d_{i}\right)} .
$$

(2) Step $M$ : the algorithm reestimates the model parameters $P(z \mid d)$ and $P(w \mid z)$ :

$$
\begin{gathered}
P\left(w_{j} \mid z_{k}\right)=\frac{\sum_{i=1}^{N} n\left(d_{i}, w_{j}\right) P\left(z_{k} \mid d_{i}, w_{j}\right)}{\sum_{j=1}^{M} \sum_{i=1}^{N} n\left(d_{i}, w_{j}\right) P\left(z_{k} \mid d_{i}, w_{j}\right),} \\
P\left(z_{k} \mid d_{i}\right)=\frac{\sum_{j=1}^{M} n\left(d_{i}, w_{j}\right) P\left(z_{k} \mid d_{i}, w_{j}\right)}{\sum_{i=1}^{N} \sum_{j=1}^{M} n\left(d_{i}, w_{j}\right)} .
\end{gathered}
$$

(3) Iteration termination condition is as follows:

After initializing $P(z \mid d)$ and $P(w \mid z)$, the EM algorithm alternately performs $E$ and $M$ steps for iterative calculation and generates new $P(z \mid d)$ and $P(w \mid z)$ until $E(L)$ reaches convergence:

$$
E(L)=\sum_{i=1}^{N} \sum_{j=1}^{M} n\left(d_{i}, w_{j}\right) \sum_{k=1}^{K} P\left(z_{k} \mid d_{i}, w_{j}\right) \log \left[P\left(w_{j} \mid z_{k}\right) P\left(z_{k} \mid d_{i}\right)\right] \text {. }
$$


PLSA is mainly divided into two processes of training and testing in image quality evaluation. Among them, in the training process, the visual words and the visual word distribution under each latent semantics are obtained by extracting features and clustering from the training image set. In the testing process, the latent semantic distribution of the test image set is compared with the latent semantic distribution of the reference image, and then the quality score of the image is obtained. The specific process is shown in Figure 2.

It can be seen from Figure 2 that the image quality score is obtained by calculating the semantic similarity of the latent semantic distribution $P\left(z \mid d_{\text {train }}\right)$ of the training image set and the latent semantic distribution $P\left(z \mid d_{\text {test }}\right)$ of the test image set. The specific process is to separately propose the latent semantic distribution of the undistorted image and calculate the average value of the latent semantic distribution according to formula (9) as a reference value for comparison. The specific process is to separately propose the latent semantic distribution of the undistorted image and calculate the average value of the latent semantic distribution according to formula (9) as a reference value for comparison. Here, $N_{p}$ is the number of reference images.

$$
P_{G}=\frac{1}{N_{P}} \sum_{i=1}^{N_{P}} P\left(z \mid d_{i}\right)
$$

There should be a certain nonlinear relationship between the subjective data and the objective data of image quality evaluation. Generally, the nonlinear function selected should be monotonous, and it is best to avoid data overfitting. Therefore, this paper uses the commonly used logarithmic regression function for fitting, and the function expression is

$$
S S_{p}=f(O S)=\frac{b_{1}}{1+\exp \left(b_{2} *\left(O S-b_{3}\right)\right)}+b_{4} * O S+b_{5} .
$$

Here, $f$ represents the nonlinear relationship, $S S_{p}$ is the fitted value, that is, the predicted subjective quality value, which is a deformation of OS, and $O S$ is the objective evaluation value. $b_{1}, b_{2}, b_{3}, b_{4}, b_{5}$ can be obtained by fitting. Figure 3 shows the prediction accuracy of the subjective MOS value, where the curve is the fitting curve of the nonlinear regression function, and the scattered points near the curve represent the data pair $\left(O S, S S_{P}\right)$.

In Figure 3, the smaller the error between $S S_{P}$ and MOS, the higher the accuracy of the algorithm. Currently, the commonly used method is taken from the performance evaluation algorithm proposed by the Video Quality Evaluation Group (VQEG). That is, the five indicators of Pearson correlation coefficient (CC), root mean square error (RMSE), average absolute error (RMSE), Spearman rank correlation coefficient (ROCC), and rate of dispersion (OR) are used as methods to evaluate the accuracy of the algorithm:

The Pearson correlation coefficient (CC) is

$$
C C=\frac{\sum_{i=1}^{n}\left(X_{i}-\bar{X}\right)\left(Y_{i}-\bar{Y}\right)}{\sqrt{\sum_{i=1}^{n}\left(X_{i}-\bar{X}\right)^{2} \sum_{i=1}^{n}\left(Y_{i}-\bar{Y}\right)^{2}}} .
$$

Here, $X_{i}$ and $Y_{i}$ are subjective and objective evaluation scores, respectively, and $\bar{X}$ and $\bar{Y}$ are, respectively, the mean value of subjective and objective evaluation.

(2) The root mean square error (RMSE) is

$$
R M S E=\sqrt{\frac{1}{n} \sum_{i=1}^{n}\left(X_{i}-Y_{i}\right)^{2}} .
$$

Here, $X_{i}$ is the subjective evaluation score, and $Y_{i}$ is the $S S_{P}$ value.

(3) The average absolute error (MAE) is

$$
M A E=\frac{1}{n} \sum_{i=1}^{n}\left|X_{i}-Y_{i}\right|
$$

Here, $X_{i}$ is the subjective evaluation score, and $Y_{i}$ is the $S S_{p}$ value.

(4) The Spearman rank correlation coefficient (ROCC) is

$$
\text { ROCC }=1-\frac{6 \sum_{i=1}^{n}\left(R X_{i}-R Y_{i}\right)^{2}}{n\left(n^{2}-1\right)} .
$$

Here, $R X_{i}$ and $R Y_{i}$, respectively, represent the data after the subjective and objective scores are sorted in the same order.

(5) The dispersion rate is

$$
\mathrm{OR}=\frac{n_{\text {owt }}}{n}
$$

Here, $n_{\text {out }}$ is the number of points where the deviation between the fitted value and the subjective value is greater than 1.5 .

In formula (11), the Pearson correlation coefficient can measure whether two data sets are on a straight line. The greater the absolute value of the correlation coefficient, the stronger the correlation. The root mean square error in formula (12) can measure the deviation between the predicted value and the subjective value. The smaller the deviation, the better the algorithm performance. The Spearman rank correlation coefficient in formula (14) can measure the monotonicity of two data sets. The larger the absolute value of ROCC, the stronger the monotonicity. The smaller the OR in formula (15), the higher the degree of aggregation of the algorithm. Therefore, the larger the value of CC and ROCC, the better the performance of the image quality evaluation algorithm. The smaller the MAE, RMSE, and OR, the worse the image quality evaluation algorithm.

Image semantics is the basic description carrier of image information content. It can convert the complete image content into an intuitively understandable text-like language expression. It is the high-level content in the field of image research and plays a vital role in the objective quality evaluation of images.

In the PLSA-based image quality evaluation, the specific application of image semantics reflects two aspects. One 


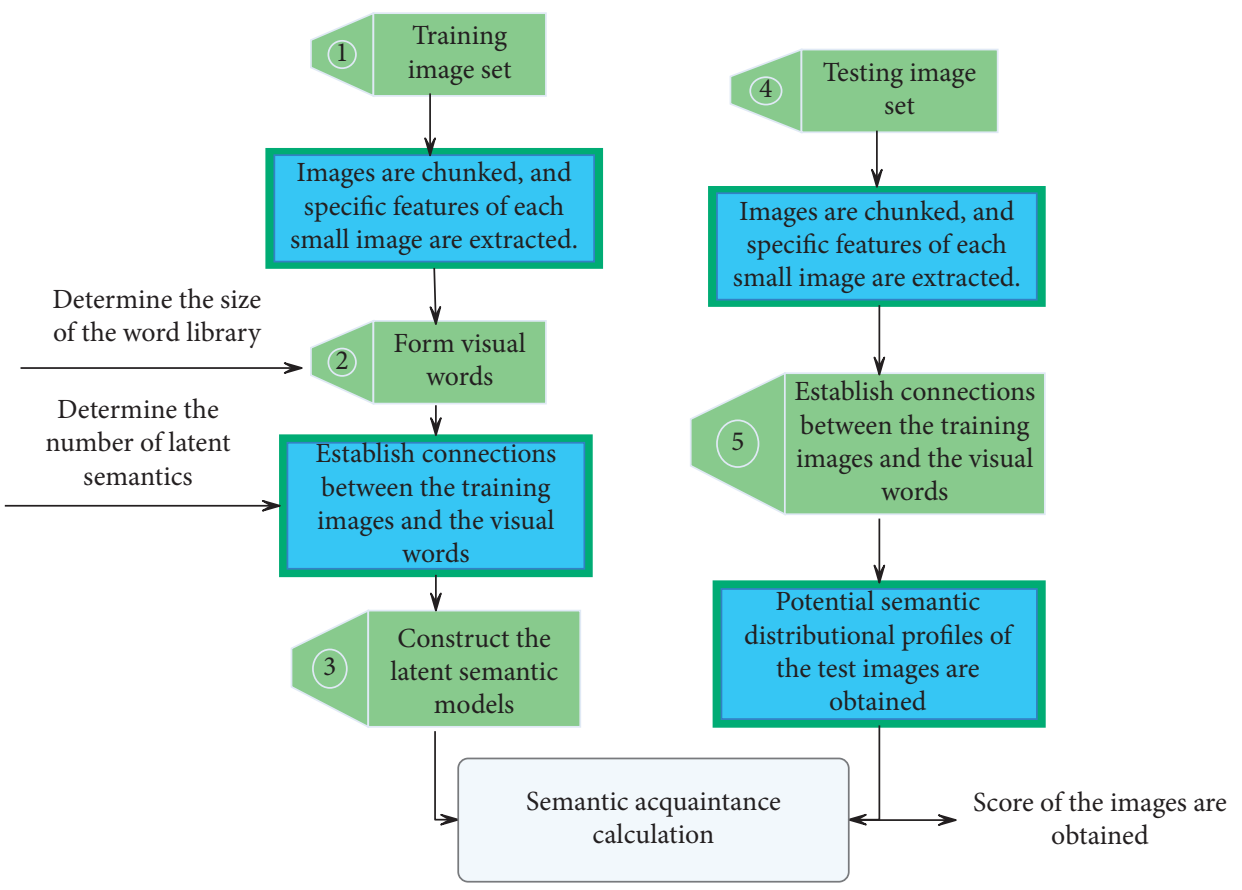

FIgURE 2: Flowchart of image quality evaluation.

aspect is the construction of the model. That is, after the lowlevel feature extraction is performed on the undistorted images and training images, the semantics are implicitly incorporated into them. This maps the high-dimensional "image-visual word" cooccurrence matrix to the low-dimensional space, thereby establishing a probability model between "image-latent semantics-visual word." On the other hand, it is reflected in the analysis of semantics, that is, the measurement of semantic similarity. Based on the rule that the latent semantic distribution of distorted images of the same type and level has similar laws, the latent semantic distribution of distorted images of different types or different levels often has certain differences. The semantic similarity measure takes the latent semantic distribution $P_{\text {pristine }}(z \mid d)$ of the undistorted image as the criterion for evaluation and comparison and then compares it with the latent semantic distribution $P_{\text {test }}(z \mid d)$ of the test image to obtain the objective score of the image. Therefore, choosing a reasonable semantic similarity measurement method is the final key step to obtain a better image quality evaluation algorithm.

For a given distorted image, this paper first calculates the normalized brightness through local mean subtraction and standardized splitting.

Ruderman believes that a local nonlinear operator can be added to the logarithmic ratio brightness to remove the local mean deviation and the standardized local variance, and there is no correlation between the two. This operator can be obtained from a given image:

$$
\widehat{I}(i, j)=\frac{I(i, j)-\mu(i, j)}{\sigma(i, j)+C} .
$$

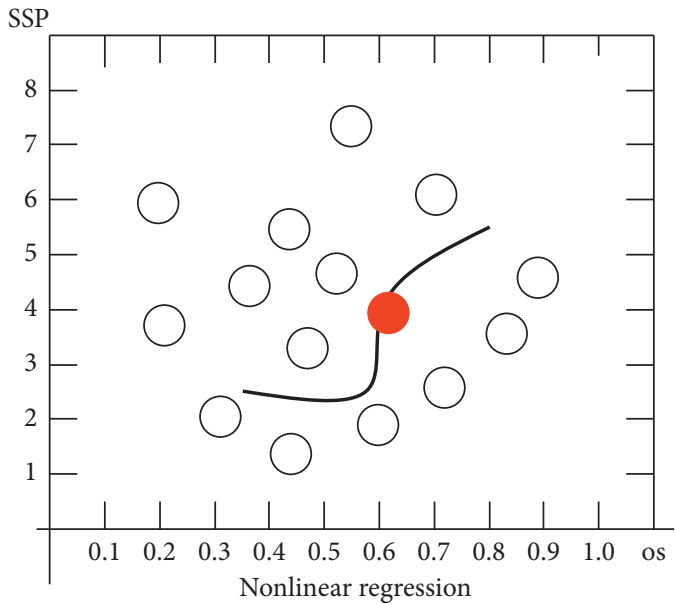

FIgURE 3: Accuracy map of subjective MOS prediction.

Here, $i \in 1,2 \ldots M, j \in 1,2 \ldots N . \mathrm{M}, \mathrm{N}$ is the height and width of the image. $C=1$ is a constant, because when the denominator approaches $0, C=1$ can prevent system instability.

$$
\begin{aligned}
& \mu(i, j)=\sum_{k=-K}^{K} \sum_{l=-L}^{L} \omega_{k, l} I_{k, l}(i, j), \\
& \sigma(i, j)=\sqrt{\sum_{k=-K}^{K} \sum_{l=-L}^{L} \omega_{k, l}\left(I_{k, l}(i, j)-\mu(i, j)\right)^{2} .}
\end{aligned}
$$

Here, $\omega=\left\{\omega_{k, l} \mid k=-K, \ldots, K, l=-L, \ldots, L\right\}$, and $\omega$ is a set of two-dimensional circularly symmetric Gaussian weight functions. 
Ruderman also observed that the normalized brightness value of natural images tends to unit Gaussian features, and this feature can be used in the contrast gain mask processing model in early human vision. In the image quality evaluation system, the brightness value $\widehat{I}(i, j)$ can be converted into MSCN (mean subtracted contrast normalized) coefficients. In the picture shown on the left picture of Figure 4, it can be seen that there is a relatively high correlation between adjacent pixels, and a diagonal structure is observed in the left picture. As shown on the right side of Figure 4, the normalization method reduces the correlation between adjacent pixels of the image.

Of course, this problem can be solved by modulating the matrix of the topic distribution probability under each image, but this also causes the score of the test image to exceed the score of the reference image. Therefore, it is unreasonable. This also shows that, to some extent, KL distance is actually not suitable as a semantic similarity algorithm for image quality evaluation. However, Hellinger distance does not have this problem. Hellinger distance is to calculate the square of the difference between $P_{G}$ and $P(z \mid I)$, so we only need to subtract the two matrices and then square each element, and we do not need to consider other factors. Therefore, Hellinger distance is more suitable for image quality evaluation than KL distance, and it can be used as a semantic similarity algorithm together with cosine similarity in image quality evaluation.

The MSCN coefficient has the characteristic of changing with the distortion, and this feature can ensure that the distortion type that affects the image quality is predicted through the image distortion change. The distribution of MSCN coefficients of blur features (blur) is closer to the Laplacian feature, while the distribution of MSCN coefficients of white noise (wn) reduces the weight of the tail of the histogram. The generalized Gaussian distribution (GGD) can effectively capture the statistical characteristics of the distorted image, and its zero-mean expression is as follows:

$$
\begin{aligned}
f\left(x ; \alpha, \sigma^{2}\right) & =\frac{\alpha}{2 \beta \Gamma(1 / \alpha)} \exp \left(-\left(\frac{|x|}{\beta}\right)^{\alpha}\right), \\
\beta & =\sigma \sqrt{\frac{\Gamma(1 / \alpha)}{\Gamma(3 / \alpha)}}, \\
\Gamma(a) & =\int_{0}^{\infty} t^{a-1} e^{-t} d t \quad a>0 .
\end{aligned}
$$

Here, $\Gamma($.$) is the gamma function. Since the coefficient$ distribution is symmetric, it can be described by the zeromean GGD distribution. Among them, the parameter $\left(\alpha, \sigma^{2}\right)$ of the GGD distribution can be fitted by the timemoment matching method.

In fact, in the GGD distribution model of the reference image and the distorted image, the parameter settings are almost the same. For each image, the MSCN coefficient can be fitted by estimating the two parameters $\left(\alpha, \sigma^{2}\right)$ in the GGD distribution model. These two parameters form the first two dimensions of the multidimensional feature and are used to capture the distortion type of the image. As for the other several-dimensional features of multidimensional features, the asymmetric generalized Gaussian distribution model can be used, and its formula is as follows:

$$
f\left(x ; v ; \sigma_{l}^{2} ; \sigma_{r}^{2}\right)=\left\{\begin{array}{l}
\frac{v}{\left(\beta_{l}+\beta_{r}\right) \Gamma(1 / v)} \exp \left(-\left(\frac{-x}{\beta_{l}}\right)^{v}\right), \\
\frac{v}{\left(\beta_{l}+\beta_{r}\right) \Gamma(1 / v)} \exp \left(-\left(\frac{-x}{\beta_{r}}\right)^{v}\right), \\
x \geq 0 .
\end{array}\right.
$$

Here,

$$
\begin{aligned}
& \beta_{l}=\sigma_{l} \sqrt{\frac{\Gamma(1 / v)}{\Gamma(3 / v)}}, \\
& \beta_{r}=\sigma_{r} \sqrt{\frac{\Gamma(1 / v)}{\Gamma(3 / v)}} .
\end{aligned}
$$

$v$ is the shape parameter of the image, which determines the distortion type of the image, and $\sigma_{l}^{2}$ and $\sigma_{r}{ }^{2}$ are the scale parameters that control the distribution of each side of the model. In fact, the AGGD model is a generalization of the GGD model. The reason is that the AGGD model is mainly for the asymmetric MSCN coefficient distribution, and the slope of the distribution is an equation about the left and right scale parameters. When $\sigma_{l}^{2}=\sigma_{r}^{2}$, the AGGD model is transformed into the GGD model. When $\eta=\left(\beta_{r}-\beta_{l}\right)(\Gamma(2 / v)) /(\Gamma(1 / v))$, the parameters $\left(\eta, v, \sigma_{l}^{2}, \sigma_{r}^{2}\right)$ of the best AGGD model can be fitted.

Due to the existence of image distortion, adjacent MSCN coefficients will show a certain "structure." Therefore, this "structure" can be obtained by calculating the statistical relationship between adjacent pixels in the four directions of the horizontal direction $(\mathrm{H})$, vertical direction $(\mathrm{V})$, main diagonal (D1), and subdiagonal (D2), as shown in Figure 4 and formula (21).

$$
\begin{aligned}
H(i, j) & =\widehat{I}(i, j) \widehat{I}(i, j+1), \\
V(i, j) & =\widehat{I}(i, j) \widehat{I}(i+1, j), \\
D 1(i, j) & =\widehat{I}(i, j) \widehat{I}(i+1, j+1), \\
D 2(i, j) & =\widehat{I}(i, j) \widehat{I}(i+1, j-1) .
\end{aligned}
$$

In this way, the abovementioned four directions are considered at the same time, and each direction is described by four parameters; that is, a total of 16 parameters constitute the 16-dimensional feature of the image.

Since human vision is multiscale, when people are observing images, the acquisition of image feature information will be interfered by scales. According to recent research on image quality evaluation, the quality evaluation algorithm combined with multiscale information can better express the correlation with human perception. Therefore, two scales of the original image and the reduced one-time image can be used to extract a total of 36-dimensional features of the image. 


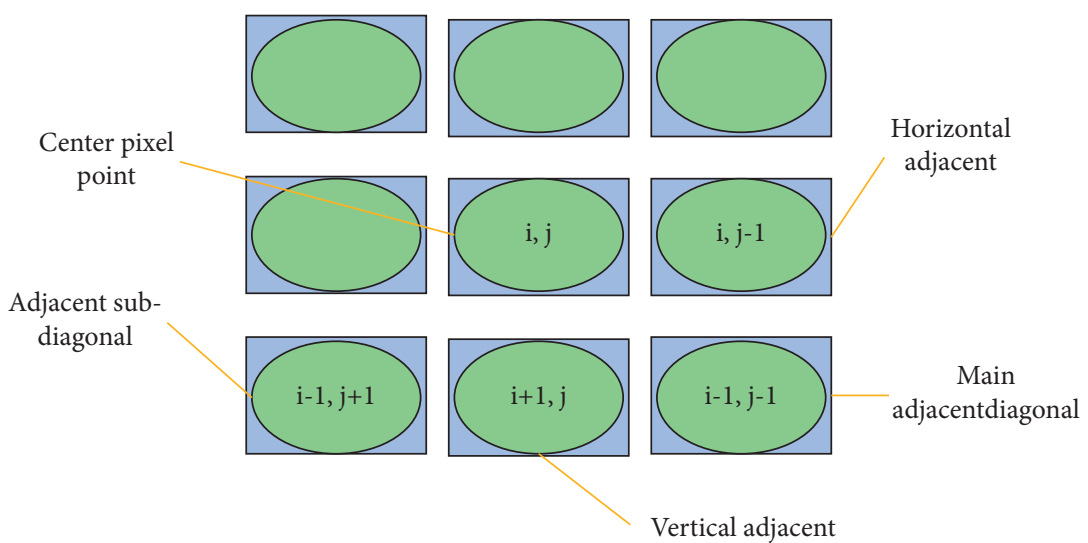

FIGURE 4: Image structure model diagram.

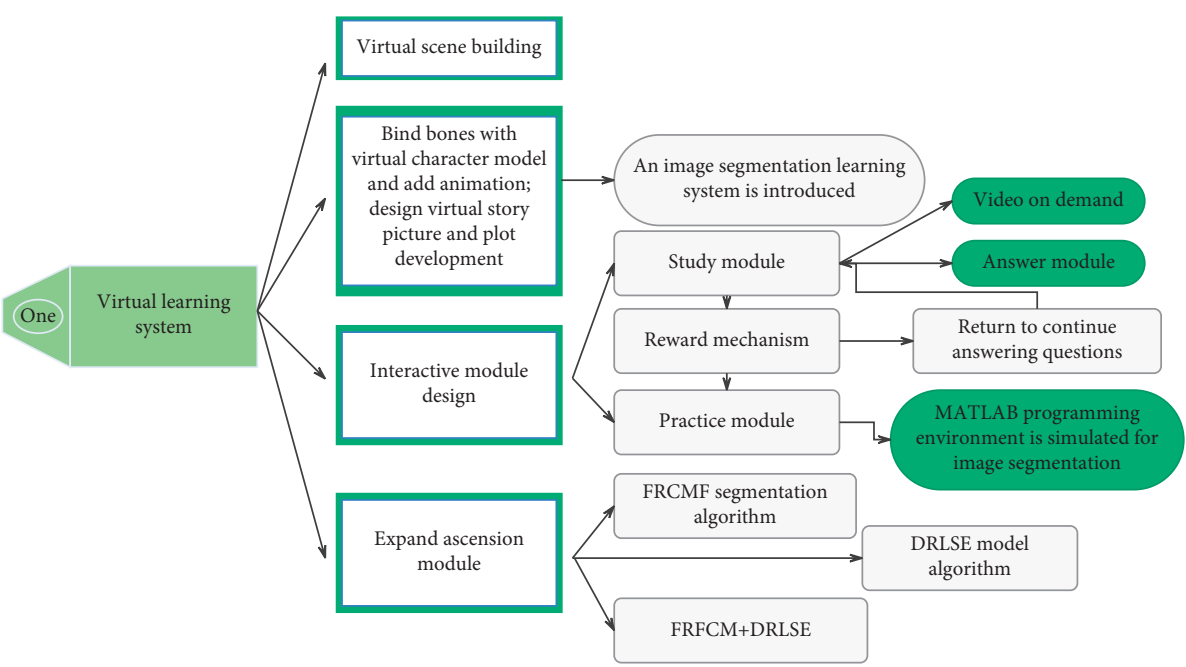

Figure 5: System technical block diagram.

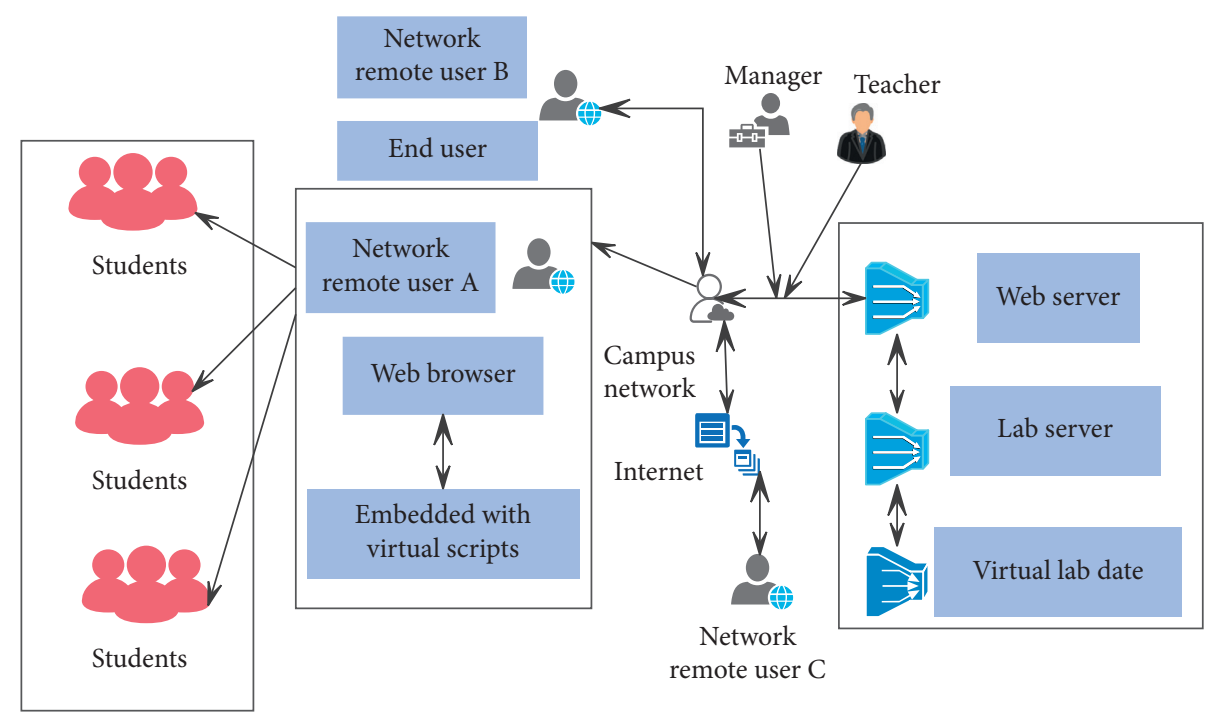

Figure 6: System overall design framework. 


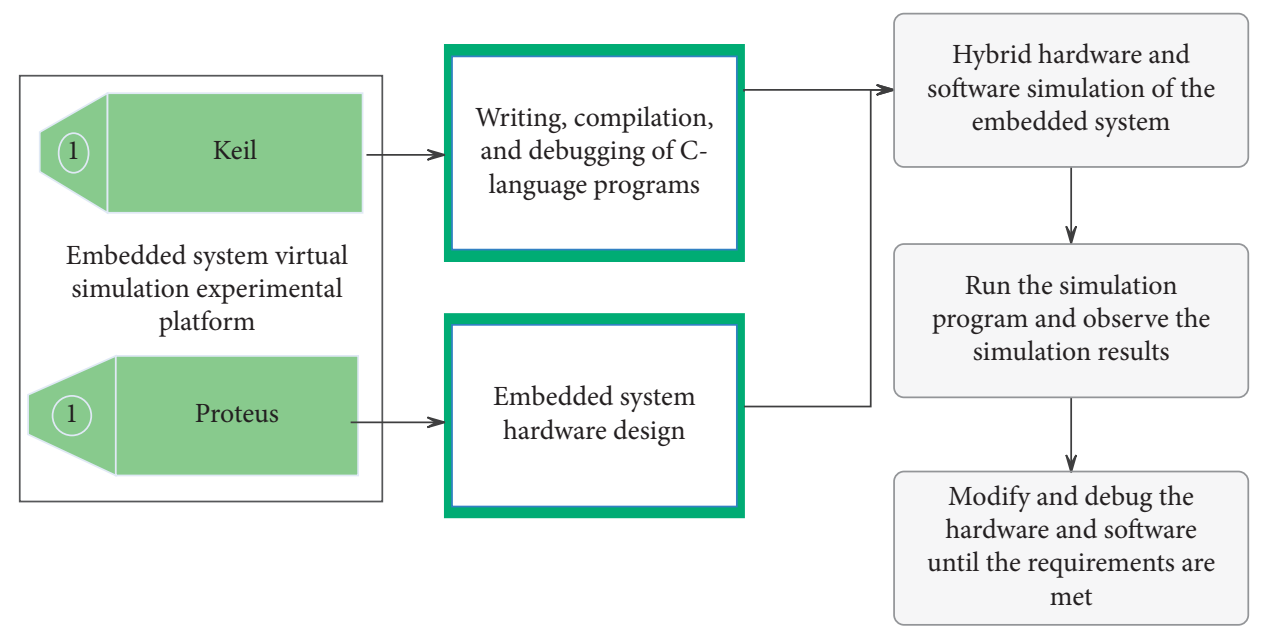

Figure 7: Virtual simulation experiment process of embedded system.

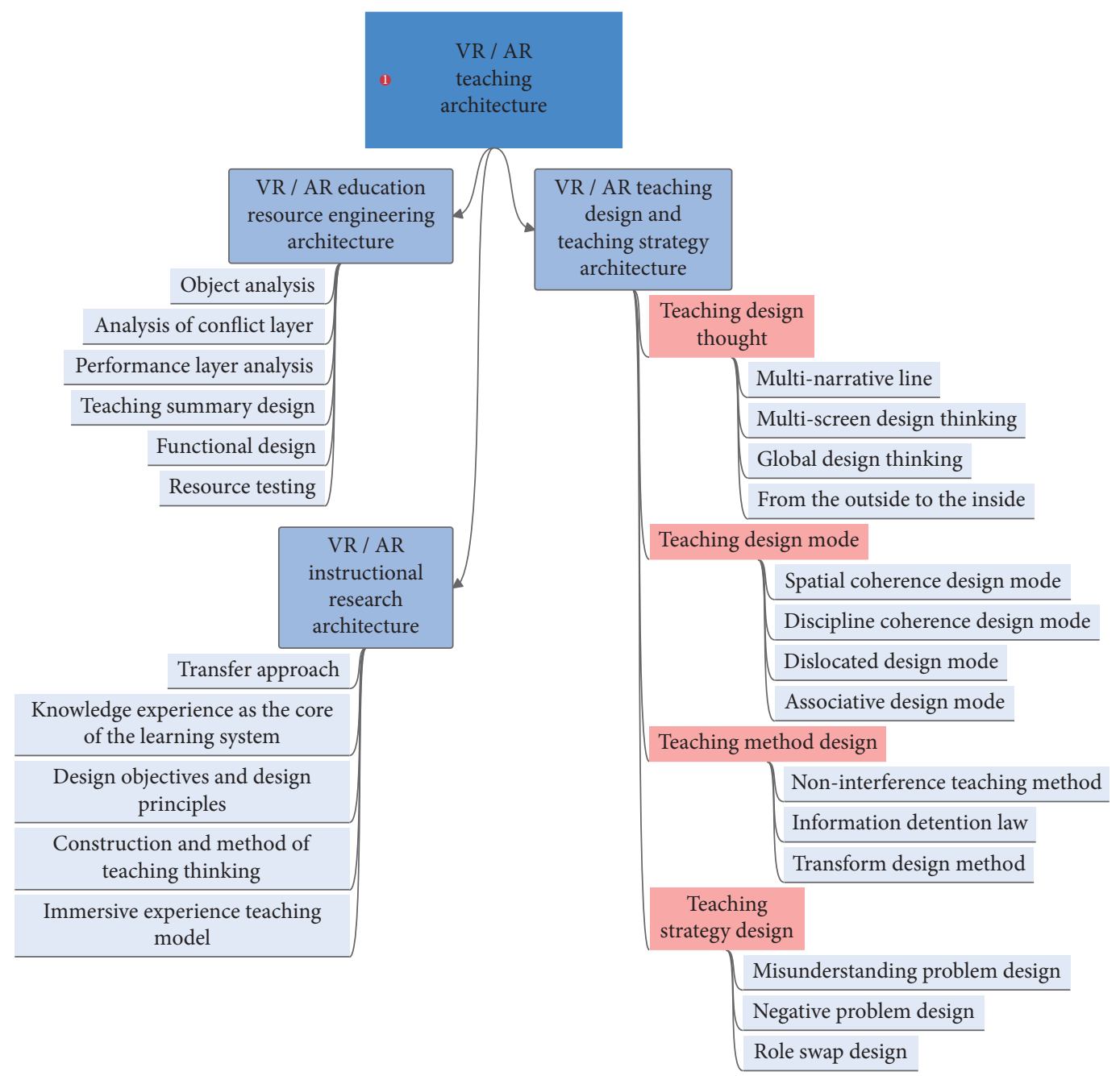

FIGURE 8: VR/AR teaching system architecture diagram. 


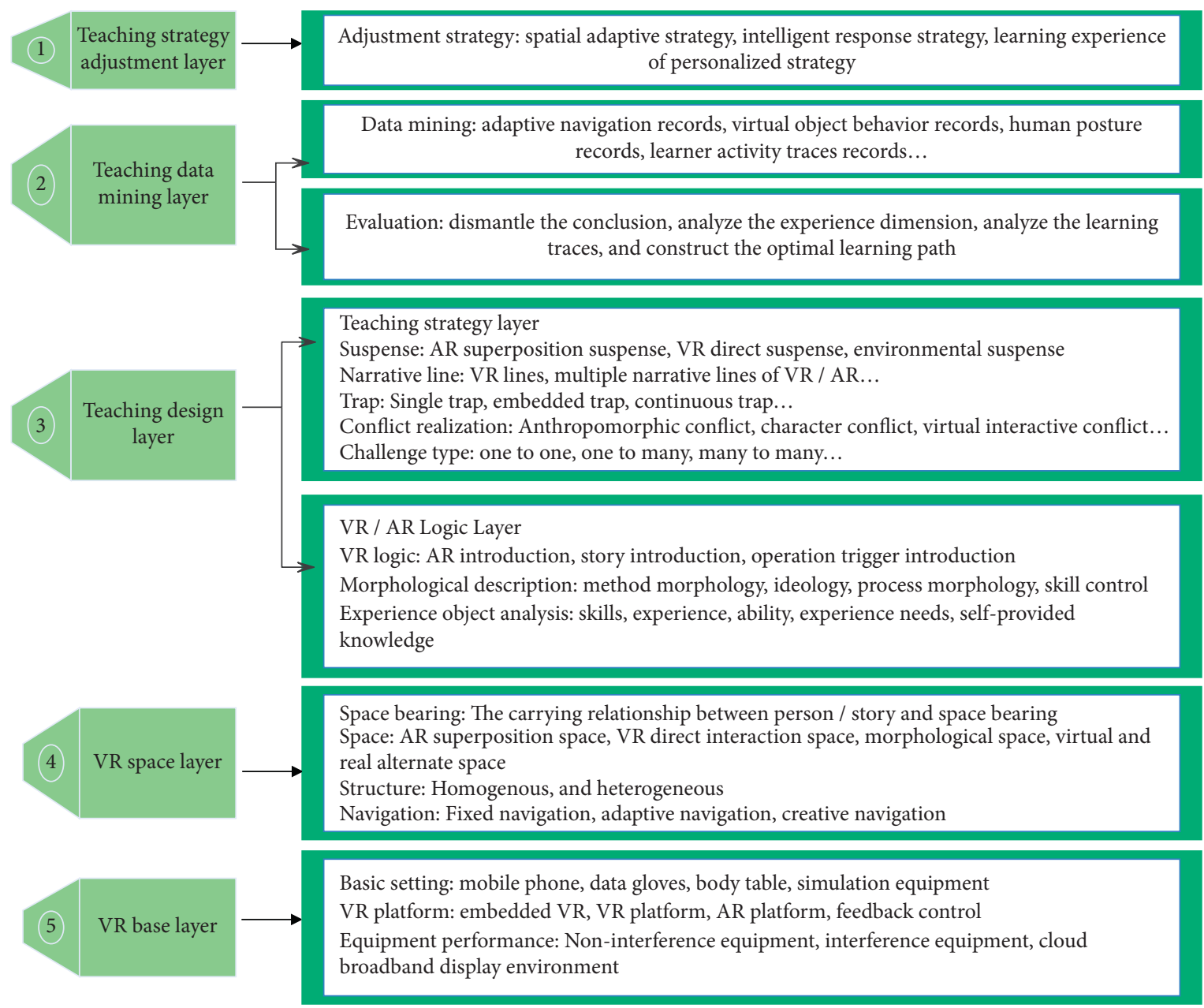

FIGURE 9: VR/AR intelligent virtual teaching control physical model.

\section{The Multimedia-Assisted Ancient Poetry Teaching System Based on AI Technology}

The learning system creates virtual characters in the first person by default, and learners roam and learn in the virtual scene according to the prompts. The overall technical block diagram of the system is shown in Figure 5.

Through the intelligent teaching system, it is helpful to mobilize students' enthusiasm and initiative in the classroom, to improve their learning efficiency, and to cultivate students' practical ability and innovation ability. The overall design framework of the system is shown in Figure 6.

This paper uses Proteus and Keil software to build an embedded system virtual simulation platform. The simulation process of 51 one-chip computer embedded system is shown as in Figure 7. First, this paper uses Proteus to draw a schematic diagram, selects Keil software to design the source code, then compiles the code, generates the target file, and then loads the HEX file in the Proteus software.

This research designs its system architecture for VR/AR teaching needs. It mainly describes the abstract components that directly constitute the VR/AR teaching system, including VR/AR teaching research architecture, VR/AR teaching design and teaching strategy framework, and VR/
AR education resource engineering framework. The specific research content is shown in Figure 8.

The AR/AR space layer contains various learning spaces of the system, including VR direct interaction space, AR superimposed space, morphological space, and virtual-real interlaced space. These spaces can be divided into four types according to their structure and properties: isomorphism and homogeneity, heterogeneity and homogeneity, isomorphism and heterogeneity, and heterogeneity and heterogeneity. In addition, the VR space also reflects the bearing relationship between people/story and space. There are four relationships between them: $1: 1,1: \mathrm{N}$, $\mathrm{N}: 1$, and $\mathrm{N}: \mathrm{N}$. It is one person and one space, one person and multiple spaces, multiple people and one space, multiple people and multiple spaces, one story and one space, one story and multiple spaces, multiple stories and one space, and multiple stories and multiple spaces. In the space, learners complete their own learning process through fixed navigation, adaptive navigation, and creative navigation, as shown in Figure 9.

This paper takes "Wanglu Mountain Waterfall" as an example to conduct multimedia-assisted research on ancient poetry teaching, and its image processing is shown in Figure 10.

Through the analysis of Figure 10, it can be seen that the multimedia-assisted artistic conception creation system of ancient poetry based on AI technology can basically meet the 


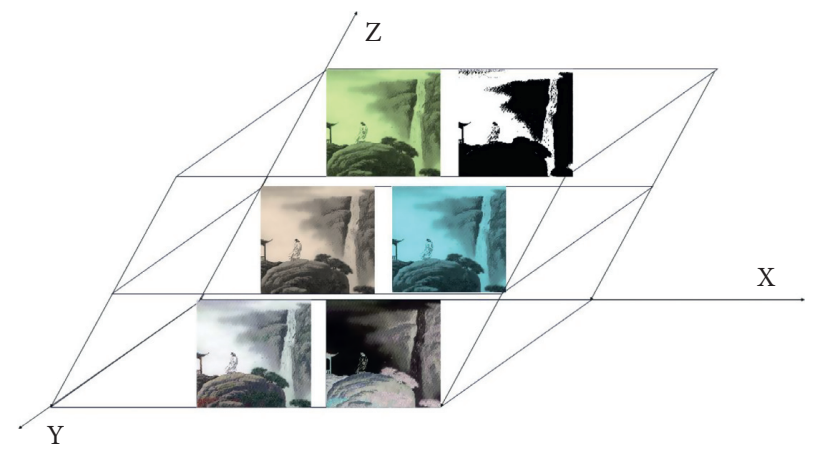

Figure 10: An example of the creation of multimedia-assisted artistic conception of ancient poetry based on AI technology.

TABLE 1: Immersive evaluation of the multimedia-assisted artistic conception creation system of ancient poetry based on AI technology.

\begin{tabular}{|c|c|c|c|c|c|}
\hline No. & Immersion & No. & Immersion & No. & Immersion \\
\hline 1 & 88.74 & 16 & 86.15 & 31 & 86.73 \\
\hline 2 & 81.99 & 17 & 90.70 & 32 & 82.53 \\
\hline 3 & 80.40 & 18 & 91.02 & 33 & 84.18 \\
\hline 4 & 91.60 & 19 & 81.61 & 34 & 91.15 \\
\hline 5 & 85.83 & 20 & 80.70 & 35 & 83.81 \\
\hline 6 & 91.07 & 21 & 90.38 & 36 & 84.85 \\
\hline 7 & 79.61 & 22 & 79.66 & 37 & 85.04 \\
\hline 8 & 82.54 & 23 & 90.31 & 38 & 87.21 \\
\hline 9 & 80.33 & 24 & 87.93 & 39 & 80.77 \\
\hline 10 & 86.91 & 25 & 88.86 & 40 & 84.74 \\
\hline 11 & 90.06 & 26 & 90.82 & 41 & 79.63 \\
\hline 12 & 84.33 & 27 & 84.07 & 42 & 80.14 \\
\hline 13 & 87.03 & 28 & 79.69 & 43 & 84.68 \\
\hline 14 & 84.10 & 29 & 87.15 & 44 & 85.51 \\
\hline 15 & 80.95 & 30 & 83.41 & 45 & 90.86 \\
\hline
\end{tabular}

TABLE 2: Evaluation of the teaching effect of the multimedia-assisted artistic conception creation system of ancient poetry based on AI technology.

\begin{tabular}{|c|c|c|c|c|c|}
\hline No. & Teaching effect & No. & Teaching effect & No. & Teaching effect \\
\hline 1 & 90.68 & 16 & 78.77 & 31 & 77.81 \\
\hline 2 & 83.74 & 17 & 85.98 & 32 & 83.36 \\
\hline 3 & 87.01 & 18 & 86.43 & 33 & 77.67 \\
\hline 4 & 89.75 & 19 & 86.96 & 34 & 84.09 \\
\hline 5 & 74.99 & 20 & 84.07 & 35 & 89.22 \\
\hline 6 & 80.11 & 21 & 80.84 & 36 & 77.38 \\
\hline 7 & 77.77 & 22 & 90.55 & 37 & 77.79 \\
\hline 8 & 79.21 & 23 & 81.58 & 38 & 80.46 \\
\hline 9 & 76.72 & 24 & 87.46 & 39 & 83.85 \\
\hline 10 & 88.31 & 25 & 90.68 & 40 & 86.53 \\
\hline 11 & 88.23 & 26 & 87.03 & 41 & 87.76 \\
\hline 12 & 82.83 & 27 & 88.66 & 42 & 78.88 \\
\hline 13 & 90.20 & 28 & 80.69 & 43 & 74.58 \\
\hline 14 & 76.42 & 29 & 85.08 & 44 & 79.20 \\
\hline 15 & 78.49 & 30 & 84.40 & 45 & 81.89 \\
\hline
\end{tabular}

teaching needs. On this basis, this paper conducts research on the performance of the system and counts the immersion and teaching effect brought by the system to students. The results are shown in Tables 1 and 2 .

From the above research, we can see that the multimedia-assisted artistic conception creation system of ancient poetry proposed in this paper has a good teaching effect and has an important role in promoting the study of the artistic conception of ancient poetry.

\section{Conclusion}

The linguistic features of ancient poems are very obvious; generally each sentence does not exceed seven characters. In a brief narrative, it either portrays a magnificent scene or a melancholy picture, which depends entirely on the author's thoughts and feelings at the time and the theme of the work itself. Therefore, ancient poems are an important form of writing in ancient China, and they are also of great 
significance in contemporary times. Many of the profound ideas still have reference significance today. This paper combines AI technology to improve the multimedia teaching mode, improve the effect of multimedia-assisted ancient poetry teaching, and lay the foundation for the subsequent improvement of the quality of ancient poetry teaching. The research shows that the multimedia-assisted artistic conception creation system of ancient poetry proposed in this paper has a good teaching effect and plays an important role in promoting the study of the artistic conception of ancient poetry.

\section{Data Availability}

The labeled datasets used to support the findings of this study are available from the corresponding author upon request.

\section{Conflicts of Interest}

The author declares no conflicts of interest.

\section{Acknowledgments}

This study was sponsored by Henan Industry and Trade Vocational College.

\section{References}

[1] S. F. M. Alfalah, "Perceptions toward adopting virtual reality as a teaching aid in information technology," Education and Information Technologies, vol. 23, no. 6, pp. 2633-2653, 2018.

[2] G. Cooper, H. Park, Z. Nasr, L. P. Thong, and R. Johnson, "Using virtual reality in the classroom: preservice teachers' perceptions of its use as a teaching and learning tool," Educational Media International, vol. 56, no. 1, pp. 1-13, 2019.

[3] J. Zhao, X. Xu, H. Jiang, and Y. Ding, "The effectiveness of virtual reality-based technology on anatomy teaching: a metaanalysis of randomized controlled studies," BMC Medical Education, vol. 20, no. 1, pp. 127-210, 2020.

[4] S. J. Bennie, K. E. Ranaghan, H. Deeks et al., "Teaching enzyme catalysis using interactive molecular dynamics in virtual reality," Journal of Chemical Education, vol. 96, no. 11, pp. 2488-2496, 2019.

[5] S. F. M. Alfalah, J. F. M. Falah, T. Alfalah, M. Elfalah, N. Muhaidat, and O. Falah, "A comparative study between a virtual reality heart anatomy system and traditional medical teaching modalities," Virtual Reality, vol. 23, no. 3, pp. 229-234, 2019.

[6] M. Reymus, A. Liebermann, and C. Diegritz, "Virtual reality: an effective tool for teaching root canal anatomy to undergraduate dental students - a preliminary study," International Endodontic Journal, vol. 53, no. 11, pp. 1581-1587, 2020.

[7] V. L. Dayarathna, S. Karam, R. Jaradat et al., "Assessment of the efficacy and effectiveness of virtual reality teaching module: a gender-based comparison," International Journal of Engineering Education, vol. 36, no. 6, pp. 1938-1955, 2020.

[8] O. Hernandez-Pozas and H. Carreon-Flores, "Teaching international business using virtual reality," Journal of Teaching in International Business, vol. 30, no. 2, pp. 196-212, 2019.

[9] V. Andrunyk, T. Shestakevych, and V. Pasichnyk, "The technology of augmented and virtual reality in teaching children with ASD," Econtechmod: Scientific Journal, vol. 7, no. 4, pp. 59-64, 2018.

[10] R. Mayne and H. Green, "Virtual reality for teaching and learning in crime scene investigation," Science \& Justice, vol. 60 , no. 5 , pp. $466-472,2020$.

[11] M. Taubert, L. Webber, T. Hamilton, M. Carr, and M. Harvey, "Virtual reality videos used in undergraduate palliative and oncology medical teaching: results of a pilot study," $B M J$ Supportive \& Palliative Care, vol. 9, no. 3, pp. 281-285, 2019.

[12] K. E. McCool, S. A. Bissett, T. L. Hill, L. A. Degernes, and E. C. Hawkins, "Evaluation of a human virtual-reality endoscopy trainer for teaching early endoscopy skills to veterinarians," Journal of Veterinary Medical Education, vol. 47, no. 1, pp. 106-116, 2020.

[13] X. Xu, P. Guo, J. Zhai, and X. Zeng, "Robotic kinematics teaching system with virtual reality, remote control and an onsite laboratory," International Journal of Mechanical Engineering Education, vol. 48, no. 3, pp. 197-220, 2020.

[14] P. W. Chang, B. C. Chen, C. E. Jones, K. Bunting, C. Chakraborti, and M. J. Kahn, "Virtual reality supplemental teaching at low-cost (VRSTL) as a medical education adjunct for increasing early patient exposure," Medical Science Educator, vol. 28, no. 1, pp. 3-4, 2018.

[15] J. Zhang and Y. Zhou, "Study on interactive teaching laboratory based on virtual reality," International Journal of Continuing Engineering Education and Life Long Learning, vol. 30, no. 3, pp. 313-326, 2020.

[16] R. Ramlogan, A. U. Niazi, R. Jin, J. Johnson, V. W. Chan, and A. Perlas, "A virtual reality simulation model of spinal ultrasound," Regional Anesthesia and Pain Medicine, vol. 42, no. 2, pp. 217-222, 2017.

[17] Y.-C. Hsu, "Exploring the learning motivation and effectiveness of applying virtual reality to high school mathematics," Universal Journal of Educational Research, vol. 8, no. 2, pp. 438-444, 2020.

[18] J. D. Anacona, E. E. Millán, and C. A. Gómez, “Aplicación de los metaversos y la realidad virtual en la enseñanza," Entreciencia e ingeniería, vol. 13, no. 25, pp. 59-67, 2019.

[19] P. Calvert, "Virtual reality as a tool for teaching library design," Education for Information, vol. 35, no. 4, pp. 439-450, 2019.

[20] J. Morimoto and F. Ponton, "Virtual reality in biology: could we become virtual naturalists?" Evolution: Education and Outreach, vol. 14, no. 1, pp. 1-13, 2021.

[21] D. Checa and A. Bustillo, "Advantages and limits of virtual reality in learning processes: briviesca in the fifteenth century," Virtual Reality, vol. 24, no. 1, pp. 151-161, 2020. 